\title{
Comparison of virulence profile of $C$. albicans \& $C$. tropicalis isolated from patients with Candida infection \& hospital environment
}

Vinaykumar Hallur ${ }^{1}$, Asoka Mohapatra ${ }^{1}$, Ashok Praharaj ${ }^{1}$, Satyajeet Mishra ${ }^{2}, \&$ Swagata Tripathy ${ }^{2}$, Departments of Microbiology ${ }^{1} \&$ Anesthesia , All India Institute of Medical Sciences , Bhubaneswar, India-751019

\section{Introduction:}

Candida albicans is the most frequent cause of invasive candidiasis(IC) worldwide. In India, IC is most commonly caused by Candida tropicalis. While, majority of infections are endogenous, exogenous infections have been described in hospitalized patients. Recently, it was demonstrated that $C$. krusei isolated from hospital environment were more virulent compared to clinical isolates. Hence, this study was done to compare the virulence factors of $C$. albicans and $C$. tropicalis isolated from different sources.

\section{Objectives:}

i)To isolate $C$. albicans \& $C$. tropicalis from hospital environment and clinical samples. ii)To determine biofilm, phospholipase and protease production of clinical and environmental isolates of $C$. albicans \& $C$. tropicalis. iii) To compare the virulence profile of clinical and environmental C. albicans \& $C$. tropicalis isolates.

\section{Methods:}

Yeasts were isolated from high touch surfaces in the hospital (medical \& surgical intensive care units, operation theatre, casualty) by swabbing sterile swabs soaked in antibiotics solution and inoculating them into brain heart infusion broth with chloramphenicol, followed by incubation at $37^{\circ} \mathrm{C}$ for 5 days. Turbid broths were then sub cultured onto $\mathrm{CHROM}$ agar and Candida identified as per standard microbiological protocol. Clinically significant yeasts submitted to diagnostic laboratory for routine culture and sensitivity during the study period were also included in this study. Ten each of $C$. albicans \& C. tropicalis from hospital environment and clinical infection were subjected to assays for biofilm formation, phospholipase, and protease production. Assays were performed in triplicate as per published protocols ${ }^{1,2}$
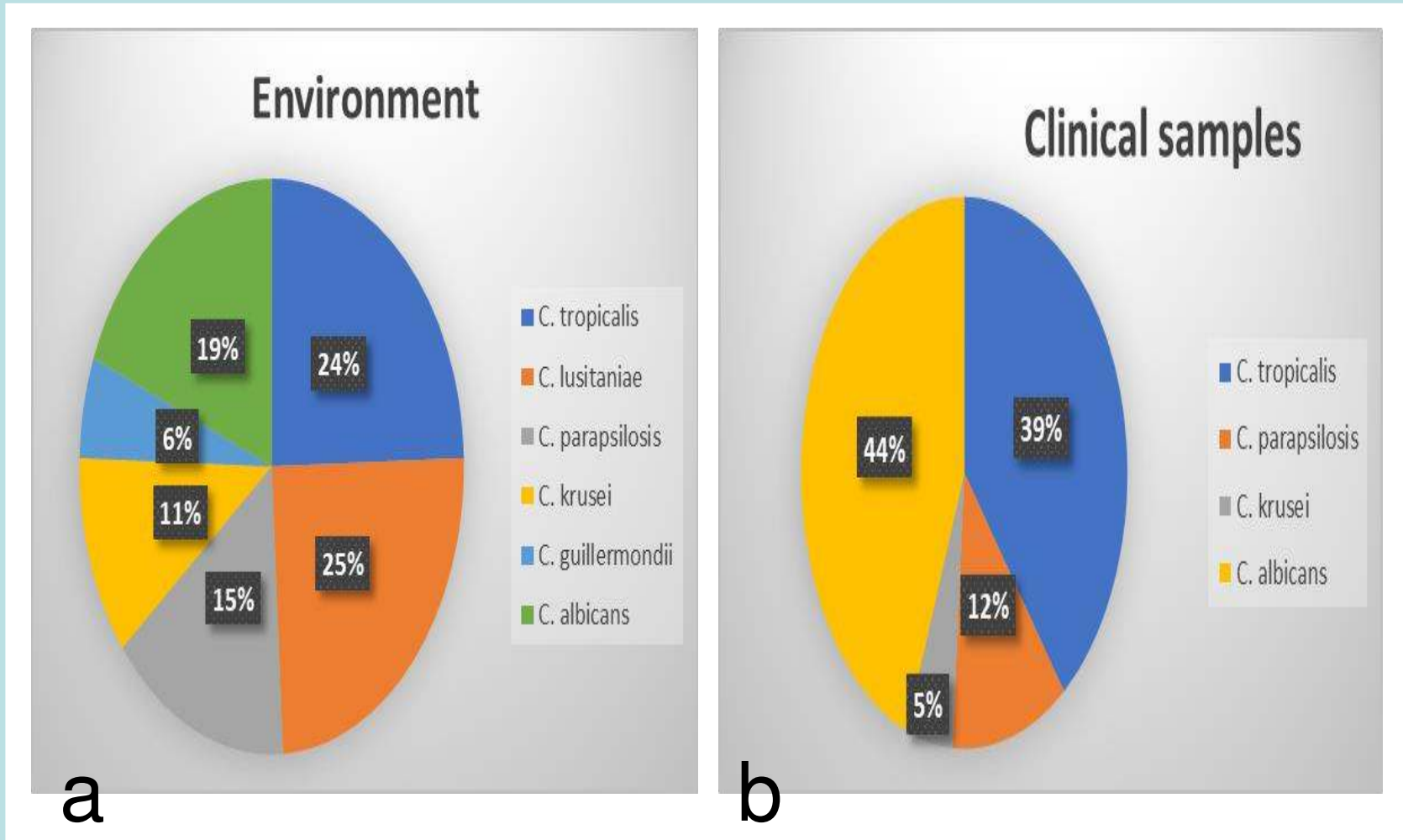

Results

\begin{tabular}{|c|c|c|c|}
\hline & $\begin{array}{c}\text { Hospital } \\
\text { environment }\end{array}$ & $\begin{array}{l}\text { Clinical } \\
\text { Samples }\end{array}$ & P value \\
\hline \multicolumn{4}{|c|}{ Biofilm(Mean $\left.A_{595}\right)$} \\
\hline C. albicans & $0.138 \pm 0.06$ & $0.128 \pm 0.08$ & 0.7 \\
\hline C. tropicalis & $0.147 \pm 0.08$ & $0.160 \pm 0.150 .6$ & 0.6 \\
\hline \multicolumn{4}{|c|}{ Phospholipase(PI index) } \\
\hline C. albicans & $7 / 10,70 \%$ & $10 / 10,100 \%$ & 0.21 \\
\hline C. tropicalis & $6 / 10,60 \%$ & $2 / 10,20 \%$ & 0.16 \\
\hline \multicolumn{4}{|c|}{ Protease(Pr index) } \\
\hline C. albicans & $0 / 10,0 \%$ & $4 / 10,40 \%$ & $0.09^{\star}$ \\
\hline C. tropicalis & $1 / 10,10 \%$ & $2 / 10,20 \%$ & 1 \\
\hline
\end{tabular}

Fifty-three Candida (26.5\%) were isolated from 200 environmental samples and 43 clinically significant isolates were obtained during the study period. Non-albicans Candida spp. (NAC) predominated (81.1\%) among environmental isolates; $C$. tropicalis $(24.52 \%)$, and C. lusitaniae $(24.52 \%)$ followed by $C$. albicans (18.9\%), C. parapsilosis (15.1\%), C. krusei (11.3\%), C. guilliermondii (5.7\%). NAC also predominated (55.8\%) in clinical isolates; C. albicans (44.2\%) followed by C. tropicalis (39.5\%), C. parapsilosis (11.6\%), C. krusei (4.7\%).No statistically significant difference in biofilm, phospholipase and proteinase production was observed between environmental \& clinical isolates of $C$. albicans \& $C$. tropicalis except more clinical $C$. albicans isolates (40\%) produced protease $(\mathrm{P}=0.09)$.

\section{Conclusion:}

No statistically significant difference in biofilm, phospholipase and proteinase production was observed between environmental \& clinical isolates except more clinical $C$. albicans isolates $(40 \%)$ produced protease $(\mathrm{P}=0.09)$. Low sample size was the biggest limitation of the study and the findings need to be confirmed using more isolates.

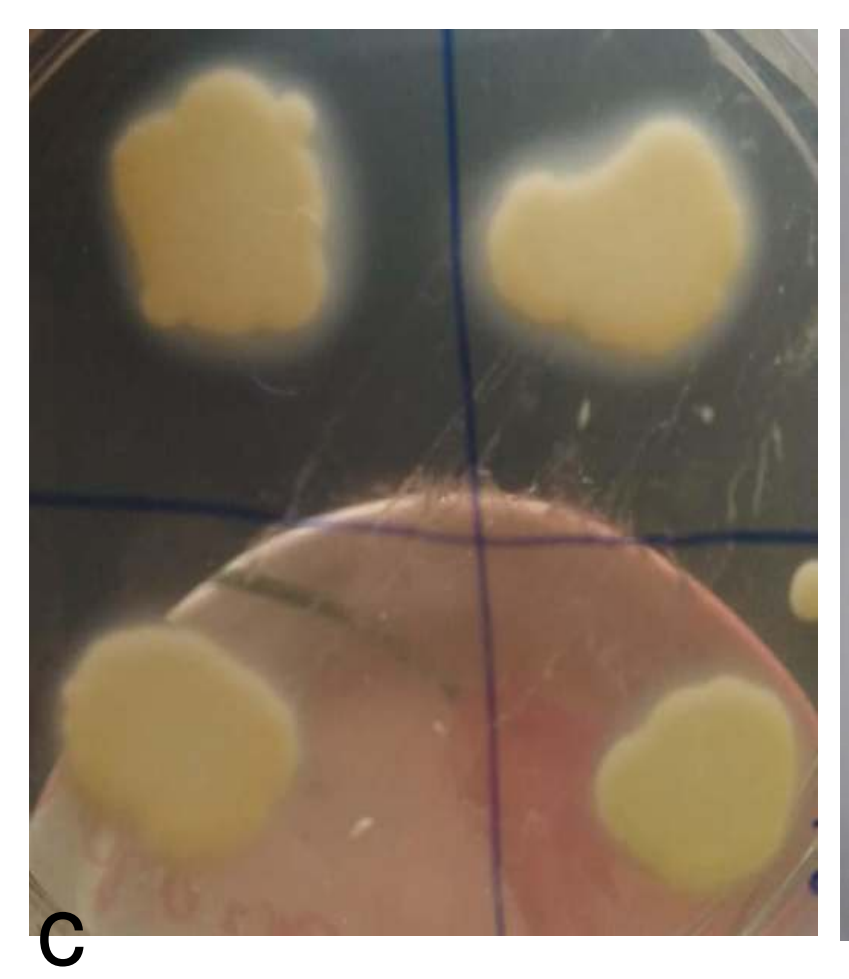

Fig from left to right a) Candida distribution in environment \& b) Clinical samples c) Phospholipase \& d) Protease assay 